\title{
A reduced-order model for porous flow through thin, structured materials.
}

\author{
Scott A. Roberts*, P. Randall Schunk* \\ Engineering Sciences Center, Sandia National Laboratories, PO Box 5800, Albuquerque, NM 87185-0836
}

\begin{abstract}
Darcy's equations are frequently used as a coarse-grained numerical expedient for modeling multiphase flow through complex porous materials. In some applications, the porous material may be quite thin, allowing the possibility of further simplification of the equations. In this paper we derive a reduced-order shell finite-element model for flow through thin porous materials using an approach similar to that taken to derive the Reynolds' lubrication equation. We advance first a formulation that addresses generalized unstructured porous materials and then specialize the equations for certain structured cases. We also extend the model to account for multiphase, confined lubrication flow in an adjoining layer and gas transport within the pores. We apply the model to several problems of topical interest in micro- and nano-manufacturing processes.

Keywords: porous media, lubrication, reduced-order model, shell element, finite element method, nano-manufacturing
\end{abstract}

\footnotetext{
${ }^{*}$ Corresponding author

Email addresses: sarober@sandia.gov (Scott A. Roberts), prschun@sandia.gov (P. Randall Schunk)
} 
velocity can be reconstructed from the gradient of the pressure field. The pressure field itself is obtained by solving a consolidated form of the continuity, or mass conservation, equation. Darcy's formulation in essence is a numerical coarse-graining approach leading to macroscopic variable descriptions of the porous flow in terms of quantities like saturation and capillary pressure. With it, simulations which utilize mesh sizes much larger than the relevant pore sizes of the underlying porous material are possible, rather than modeling flow through individual pores. An excellent monograph of flow through porous media and its basic equations was published by Bear [1], and we defer to this work to review relevant literature concerning flow in porous media.

The Darcy formulation is in general applicable to porous material domains where the representative volume elements used in the simulations are larger than the characteristic pore dimensions. However, an exception to this is when the porous material dimension in one direction is much thinner than the other two, such as would appear in thin porous sheets. In this case, macroscopic properties of the pores, such as permeability, become anisotropic. A computational expedient that is useful for such material domains is a reduced-order, finiteelement approach utilizing shell elements. Shell element technology is widely used in the solid mechanics community, specifically as a more efficient and often more accurate approach to model beams, bars, and membranes $[7,8]$. In contrast, computational shell elements are uncommon in the fluid mechanics community, despite the abundance of reduced-order models for fluid flow, including lubrication flow [9] and the thin-film flow equations [10]. Recently, Roberts et al. [11] demonstrated the implementation of the lubrication equations using shell elements, extending the traditional model to incorporate multiphase flow using a level-set method.

In this paper, we expand on the work of Roberts et al. [11], deriving a reduced-order formulation for multiphase flow through thin porous media and implementing it in a finite element method using shell elements. The porous microstructures in this model are taken as unstructured, as often occurs in non-woven products, such as paper, or structured, as is often found in manufacturing applications. While this model is derived generally and is applicable to a wide range of multiphase flow applications, our work is primarily motivated by the nano-manufacturing industry, specifically the step-and-flash imprint lithography (SFIL) 


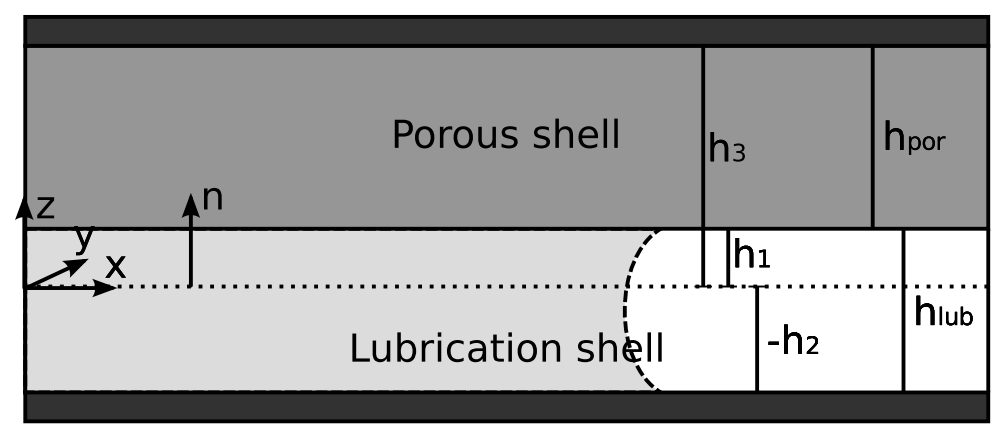

Figure 1: Illustration of the geometry used in this model. The system is bounded at the bottom by a rigid, impermeable substrate, shown in black. Atop this substrate is a multiphase lubrication layer of thickness $h_{\text {lub }}$, where the light gray region represents a liquid and the white region is a gas. The liquid-air interface is shown with the dashed curve. This lubrication layer is bounded above by a porous medium of thickness $h_{\text {por }}$. The location of these material boundaries are denoted by $h_{i}$, such that $h_{\text {lub }}=h_{1}-h_{2}$ and $h_{\text {por }}=h_{3}-h_{1}$.

process [12]. Previously, flow into individual nanopores has received two-dimensional (2D) and three-dimensional (3-D) treatment from the continuum standpoint [13-17]. More recently, Chauhan et al. [18] took the approach of creating a simple coarse-grained model that assumed an axisymmetric liquid drop is being squeezed under a homogeneous template of features, studying the rate of liquid flow and feature filling. This approach is the most similar to what we propose in this work.

This paper is organized as follows. In $\S 2$, we survey the lubrication formulation $(\S 2.1)$ and derive the reduced-order equations for flow through porous media ( $(2.2)$, and then specialize them for two different pore structure types $(\S 2.2 .1, \S 2.2 .3)$. The numerical implementation of these models is discussed in $\S 3$. These models are numerically demonstrated in $\S 4$ using five example problems. Finally, we will draw conclusions on the use of these models and discuss future directions in $\S 5$.

\section{Model development}

In this section, we derive the governing equations for flow in a thin porous medium and further present the associated equations and proposed couplings for multiphase lubrication flows. The geometry used for this development is shown in figure 1. This system is characterized by physical properties and parameters which will be discussed further in the following sub-sections. All quantities, including the thickness of the layers, may vary spatially and 
temporally, as $\beta=\beta(x, y, t)$, to represent non-uniform topology and/or spatially inhomogeneous materials. The horizontal (normal to $z$ ) boundaries shown in figure 1 are free to move due to the response of an applied force, as will be shown later in this work. Only a small portion of the $x$ and $y$ directions are shown here, and the domain is considered to be much larger.

In figure 1 , the substrate is shown aligned in the $x-y$ plane, an arbitrary choice made for ease of the presentation of equations. However, our formulation can be generalized to accommodate arbitrary orientation consistent with previous work [11]. Specifically, generalized curvilinear coordinates are accommodated through the use of the shell gradient operator, $\nabla_{\|}=(\boldsymbol{I}-\boldsymbol{n n}) \cdot \boldsymbol{\nabla}$, where $\boldsymbol{I}$ is the identity tensor, $\boldsymbol{n}$ is the normal vector to the shell/substrate, illustrated in figure 1 , and $\boldsymbol{\nabla}$ is the traditional three-dimensional gradient operator. Additional implementation details are discussed by Roberts et al. [11].

\subsection{Multiphase lubrication flow}

The source and sink for pore liquid in the applications of interest arise the gas and liquid presence/inventory in an adjoining fluid phase. A multiphase lubrication model governs the mass and momentum balance in this layer and was presented thoroughly by Roberts et al. [11]. We summarize that model here for completeness.

Flow in a thin region is governed by the lubrication theory of Reynolds [19], and is stated by the governing equation

$$
-\boldsymbol{\nabla}_{\|} \cdot \boldsymbol{q}_{\mathrm{lub}}+\boldsymbol{B}_{1, \|} \cdot \boldsymbol{\nabla}_{\|} h_{\mathrm{lub}, 1}-\boldsymbol{B}_{2, \|} \cdot \boldsymbol{\nabla}_{\|} h_{\mathrm{lub}, 2}=\boldsymbol{n} \cdot\left(\boldsymbol{B}_{1, \mathrm{n}}-\boldsymbol{B}_{2, \mathrm{n}}\right) .
$$

In this equation, $\boldsymbol{B}_{i}$ is the translation of boundary $i$, where $i=2$ is the bottom, rigid substrate and $i=1$ is the top, porous medium. Tangential movement of the boundary is given by $\boldsymbol{B}_{i, \|}=(\boldsymbol{I}-\boldsymbol{n n}) \cdot \boldsymbol{B}_{i}$ and movement or flow normal to the boundary is $\boldsymbol{B}_{i, \mathrm{n}}=\boldsymbol{n n} \cdot \boldsymbol{B}_{i}$. It is important to note that flow through the upper, porous boundary is included in the $\boldsymbol{B}_{1, \mathrm{n}}$ term. Gradients in the inclination of the upper or lower boundaries are expressed by $\nabla_{\|} h_{\mathrm{lub}, i}$. The primary physics of this equation are contained in the flow rate term, $\boldsymbol{q}_{\text {lub }}$, which is given by

$$
\boldsymbol{q}_{\text {lub }}=-\frac{h_{\text {lub }}^{3}}{12 \mu}\left(\boldsymbol{\nabla}_{\|} p_{\text {lub }}-\rho \boldsymbol{g}_{\|}+\boldsymbol{F}_{\mathrm{CSF}}\right)+\frac{h_{\mathrm{lub}}}{2}\left(\boldsymbol{B}_{1, \|}+\boldsymbol{B}_{2, \|}\right)
$$


The independent variable in this system is the lubrication pressure, $p_{\text {lub. Additionally, } \mu \text { is }}$ the fluid viscosity, $\boldsymbol{g}_{\|}=(\boldsymbol{I}-\boldsymbol{n n}) \cdot \boldsymbol{g}$ is the gravity vector, and $\boldsymbol{F}_{\mathrm{CSF}}$ is the surface tension body force, with other variables defined in figure 1. A mean velocity field can be calculated from $\boldsymbol{q}_{\text {lub }}$ by $\overline{\boldsymbol{u}}=\boldsymbol{q}_{\text {lub }} / h_{\text {lub }}$.

Interfaces between two fluids (in this case, a liquid and air, as shown in figure 1) are captured with a level-set field [20]. The level-set distance function, $f$, is governed by

$$
\frac{\partial f}{\partial t}=-\overline{\boldsymbol{u}} \cdot\left|\boldsymbol{\nabla}_{\|} f\right| .
$$

Surface tension forces in the momentum balance are calculated from this level-set field using the balanced-force formulation [11, 21, 22]. Specifically, the force can be computed from the curvature as

$$
\boldsymbol{F}_{\mathrm{CSF}}=\sigma \kappa \boldsymbol{\nabla}_{\|} H \text {. }
$$

Here, $\sigma$ is the surface tension, $\kappa$ is the total curvature of the interface, and $H$ is the smoothed Heaviside function describing the location of the interface. The curvature and Heaviside function are determined from the level-set field. Specifics of this implementation can be found in Roberts et al. [11]. All material properties are also weighted by the smoothed Heaviside function, following

$$
\beta=\beta_{\mathrm{a}}+\left(\beta_{\mathrm{b}}-\beta_{\mathrm{a}}\right) H,
$$

where $\beta$ is a generic material property and the $a$ and $b$ indices represent the properties for phase $a$ and $b$, respectively.

The total curvature $\kappa$ is broken down into two components, $\kappa=\kappa_{\mathrm{n}}+\kappa_{\|}$. The curvature in the transverse direction is calculated from the level-set field,

$$
\kappa_{\|}=\nabla_{\|} \cdot \boldsymbol{n}_{a b},
$$

while the curvature through the lubrication thickness is given by the model

$$
\kappa_{z}=\frac{1}{h_{\mathrm{lub}}}\left[\cos \left(\pi-\theta_{1}-\tan ^{-1}\left(\boldsymbol{n}_{a b} \cdot \nabla_{\|} h_{\mathrm{lub}, 1}\right)\right)+\cos \left(\pi-\theta_{2}-\tan ^{-1}\left(\boldsymbol{n}_{a b} \cdot \nabla_{\|} h_{\mathrm{lub}, 2}\right)\right)\right],
$$

which assumes a circular arc for the interface, as shown in the profile view in figure 1 . In these equations, $\boldsymbol{n}_{a b}=\nabla_{\|} f$ is the normal vector to the interface and $\theta_{i}$ is the liquid contact angle at boundary $i$. 


\subsection{Thin-region porous flow}

Flow through porous media has been modeled for over a century using the Darcy formulation [2]. Darcy's law relates a spatially-averaged liquid pressure, $p_{\text {por }}$, to the liquid flux, or superficial velocity, $\boldsymbol{v}$,

$$
\boldsymbol{v}=-\frac{1}{\mu} \boldsymbol{K}_{\mathrm{eff}} \cdot \boldsymbol{\nabla} p_{\mathrm{por}} .
$$

The effective permeability, $\boldsymbol{K}_{\text {eff }}$, is cast as a tensor to accommodate anisotropies in the thin porous structure. For multi-phase flow, this permeability is often represented by a combination of the intrinsic permeability, $\boldsymbol{K}$, which is determined by the structure of the porous microstructure, and a relative permeability multiplier, $k_{\text {rel }}$, which is used to adjust for the pore space available to flow due to partial saturation, such that $\boldsymbol{K}_{\text {eff }}=k_{\text {rel }} \boldsymbol{K}$. The relative permeability is clearly a function of saturation and is commonly expressed with models such as that posed by van Genuchten [23], which is a function of the saturation of the pore space, $S$. For isotropic materials, the intrinsic permeability (or simply the permeability throughout this paper) can be characterized by a scalar $k, \boldsymbol{K}=k \boldsymbol{I}$. An unsteady-state mass conservation equation completes the description of this flow,

$$
-\frac{\partial C}{\partial t}=\rho \boldsymbol{\nabla} \cdot \boldsymbol{v},
$$

where $C$ is the liquid mass concentration in the medium and $\rho$ is the liquid density. It is common to recast (9) in terms of saturation:

$$
-\phi \frac{\partial S}{\partial t}=\boldsymbol{\nabla} \cdot \boldsymbol{v} .
$$

Here, $\phi$ is the porosity of the material, which is the ratio of the volume of pore space to the total volume, and $S$ is the saturation of the material, defined as the ratio of the volume of pore space filled with liquid to the total pore volume. Clearly, if we take $\rho$ and $\phi$ as constant in time, $C=\rho \phi S$.

Like the lubrication-flow layer, the porous layer is thin. Additionally, the microstructure and hence the corresponding porous properties are often anisotropic, by virtue of the way the medium is manufactured. For model efficiency we seek a reduced-order model similar to that of $\S 2.1$ which takes advantage of these features. The velocity can be separated into 
components normal $\left(\boldsymbol{v}_{\mathrm{n}}\right)$ and tangential $\left(\boldsymbol{v}_{\|}\right)$to the transverse direction,

$$
\boldsymbol{v}=(\boldsymbol{I}-\boldsymbol{n n}) \cdot \boldsymbol{v}+\boldsymbol{n n} \cdot \boldsymbol{v}=\boldsymbol{v}_{\|}+\boldsymbol{v}_{\mathrm{n}}
$$

Combining (10) with (11) gives

$$
-\phi \frac{\partial S}{\partial t}=\nabla_{\|} \cdot \boldsymbol{v}_{\|}+\nabla_{\mathrm{n}} \cdot \boldsymbol{v}_{\mathrm{n}}
$$

If we make the assumption, typical of a lubrication-type model, that gradients of velocity do not change through the thickness of the material, then (12) can be integrated through the thickness of the film to obtain

$$
-h_{\mathrm{por}} \phi \frac{\partial S}{\partial t}=h_{\mathrm{por}} \boldsymbol{\nabla}_{\|} \cdot \boldsymbol{v}_{\|}+\left.\boldsymbol{n} \cdot \boldsymbol{v}_{\mathrm{n}}\right|_{z=h_{3}}-\left.\boldsymbol{n} \cdot \boldsymbol{v}_{\mathrm{n}}\right|_{z=h_{1}} \text {. }
$$

At the top of the porous layer we assume no flux,

$$
-h_{\text {por }} \phi \frac{\partial S}{\partial t}=h_{\text {por }} \nabla_{\|} \cdot \boldsymbol{v}_{\|}-\left.\boldsymbol{n} \cdot \boldsymbol{v}_{\mathrm{n}}\right|_{z=h_{1}}
$$

In a similar fashion, we decompose (8), into normal-tangential components and combine with (14) to give

$$
-h_{\text {por }} \phi \frac{\partial S}{\partial t}=-\frac{h_{\text {por }}}{\mu} \nabla_{\|} \cdot\left(\boldsymbol{K}_{\|} \cdot \nabla_{\|} p_{\text {por }}\right)+\left.\frac{1}{\mu} \boldsymbol{K}_{\mathrm{n}} \nabla_{\mathrm{n}} p_{\text {por }}\right|_{z=h_{1}},
$$

where $\boldsymbol{K}_{\|}=(\boldsymbol{I}-\boldsymbol{n n}) \cdot \boldsymbol{K}_{\mathrm{eff}}$ and $\boldsymbol{K}_{\mathrm{n}}=\boldsymbol{n \boldsymbol { n }} \cdot \boldsymbol{K}_{\mathrm{eff}}$. In (15) the first term accounts for the accumulation of liquid in the pore space (or saturation of the pores), the second term accounts for pressure-driven flow of liquid through the transverse direction of the porous medium, and the third term accounts for any exchange flux of liquid with the adjacent lubrication layer. As was necessary for the curvature in the $z$ direction in $\S 2.1$, a model is required for this final source term, the form of which depends on the pore structure.

This formulation assumes that as a liquid front moves through the lubrication layer, it instantaneously passes over a pore, trapping gas in the pore, rather than the meniscus wetting through the pore. This behavior is applicable when the aspect ratio of the pore (defined as the pore height over the pore diameter) is large and the wetting front moves at a significantly fast speed, as was studied by Reddy et al. [14]. In the applications of interest to us, the interface moves at sufficiently fast speeds that all of the uptake occurs after the 


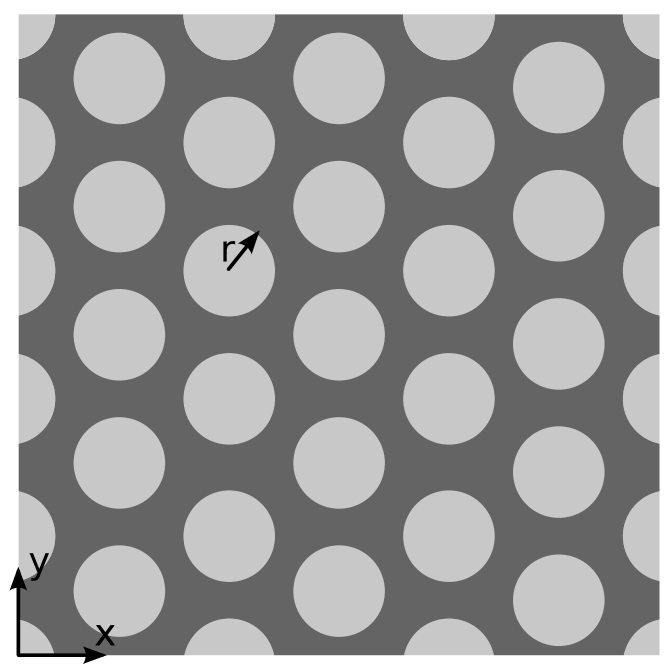

Figure 2: Sample illustration of a closed-feature porous material, shown in the $x-y$ plane, featuring an array of hexagonal-packed holes in an otherwise impermeable solid. Light gray regions are pores while the dark gray is impermeable material.

surface is wetted and does not invade the pore during lateral distribution in the lubrication gap.

We recognize that there are numerous extensions to the simple version of Darcy's law presented here. The unsteady formulation for Darcy's law is used to remove short-time singularities, but is typically not necessary for flows on time scales greater than a nanosecond [24]. Brinkman's extension can be used to account for transitional flow between boundaries [25]. While either of these extensions could be included in this shell formulation, we have chosen to neglect them for simplicity of the presentation.

\subsubsection{Closed-feature flow}

The manufacturing applications motivating the development of this model involve flows through highly structured porous regions. An example of this type of geometry is shown on the feature scale in figure 2 , in which a thin solid sheet partially perforated with a regular array of holes is treated in our model as a thin porous medium. Each of these pores is cylindrical having radius $r$ and height $h_{\text {por }}$. They are open to the lubrication layer at $z=h_{1}$ but closed at the top, $z=h_{3}$. Each of these pores is isolated and liquid cannot flow between them, except by flowing through the adjacent lubrication layer. That is, each of these pores, or features, is "closed." In this geometry, we assume each pore is initially filled with an 
ideal gas at a specified pressure and the invading fluid is liquid. This assumption of onedimensional flow is a simplification to the models, relevant for this specific geometry, and will be generalized to three-dimensional flow in the following sections.

With no lateral flow in the porous medium, significant simplifications of (15) can be made. Specifically, the first term on the right-hand side vanishes, leaving

$$
-h_{\text {por }} \phi \frac{\partial S}{\partial t}=\left.\frac{1}{\mu} K_{z z} \frac{d p_{\text {por }}}{d z}\right|_{z=h_{1}} .
$$

Here, the last term was simplified by recognizing that there is only one remaining component to the permeability tensor, $K_{z z}$, and that the normal component of the pressure gradient is in the $z$ direction. Darcy's law (8) suggests a simple expression for the velocity in the $z$ direction,

$$
v_{z}=-\frac{1}{\mu} K_{z z} \frac{d p_{\mathrm{por}}}{d z} .
$$

To model this geometry using a continuum porous flow equation such as (16), we must upscale our microscopic view of the geometry shown in figure 2 and create continuum properties from it. In this case, an expression for the permeability and pressure gradient must be determined.

From the Hagen-Poiseuille law for flow through a cylindrical pipe [26], we derive an expression for the mean velocity as a function of the pressure gradient.

$$
v_{\text {pipe }}=-\frac{r^{2}}{8 \mu} \frac{d p}{d z} .
$$

To account for an array of cylinders with porosity $\phi$, we multiply (18) by $\phi$ to obtain the mean velocity through an array of cylindrical holes, $v_{z}=\phi v_{\text {pipe }}$. Comparing (17) and (18) leads to a simple form for the permeability, $K_{z z}=\phi r^{2} / 8$.

This procedure for determining the permeability is easily generalizable to other closed pore configurations oriented in the $z$ direction. For example, following the solution for laminar flow in a square duct presented by Panton [27], one can integrate the velocity profile to determine the mean velocity and solve for the effective permeability, $K_{z z}^{\text {square }}=$ $0.0351449 L^{2} \phi$, where $L$ is the half-width of the square channel, $L$ being the half-width of the channel.

In this reduced-order model, pressure gradients in the $z$ direction are not resolved numerically (i.e., not resolved with mesh points). Therefore, a model is necessary for $\frac{d p_{\text {por }}}{d z}$ in 
(16). We assume that the pressure gradient can be approximated with a finite difference over $\Delta z$, the distance into the pore penetrated by liquid $\left(S h_{\mathrm{por}}\right)$. The pressure difference over this distance can be calculated by knowing the pressure in the lubrication layer (which is assumed to be equal to the pressure at the bottom or the pore) and the pressure below the interface $\left(z=h_{1}+S h_{\mathrm{por}}\right)$,

$$
\begin{aligned}
\frac{d p_{\text {por }}}{d z} & =\frac{\Delta p_{\text {por }}}{\Delta z} \\
& =\frac{\left.p\right|_{z=h_{1}+S h_{\text {por }}}-\left.p\right|_{z=h_{1}}}{S h_{\text {por }}} \\
& =\frac{\left(p_{\text {gas }}-p_{\text {cap }}\right)-p_{\text {lub }}}{S h_{\text {por }}}
\end{aligned}
$$

Here, $p_{\text {cap }}$ is the capillary pressure arising from the presence of a gas-liquid interface. In the last line of (19), we recognize that the pore is initially full of gas at atmospheric pressure. When liquid invades the pores, the gas is compressed and its pressure increases. The pressure below the liquid interface is simply adjusted by the capillary pressure jump across the interface.

We determine the pressure in the trapped gas from the ideal gas law. Assuming that the system is isothermal, this yields the expression $p_{\text {por }} V_{\text {por }} / n=p_{\text {por }}^{0} V_{\text {por }}^{0} / n^{0}$. Here, $n$ is the moles of gas in the pore, $V_{\text {por }}$ is the volume of the pore occupied by gas, and a superscript ${ }^{0}$ denotes the initial condition. Because the saturation is inversely proportional to the gas volume, we relate the current and initial volume, $V_{\mathrm{por}} / V_{\mathrm{por}}^{0}=1-S$. Defining a molar change ratio as $\bar{n}=n / n^{0}$, we can express the gas pressure as

$$
p_{\text {gas }}=\frac{p_{\text {gas }}^{0} \bar{n}}{1-S} \text {. }
$$

In the simplest case, all of the gas is trapped in the pore, so $\bar{n}=1$. We relax this assumption in $\S 2.2 .2$ and allow gas to diffuse out of the pores and into the liquid, leading to $\bar{n}<1$.

The jump in pressure across the liquid-gas interface, or capillary pressure $p_{\text {cap }}$, is calculated from the Young-Laplace equation,

$$
p_{\text {cap }}=\frac{2 \sigma \cos \left(\theta_{1}\right)}{r} .
$$

Traditionally, as in (7), the contact angle $\theta_{1}$ is taken as the equilibrium, or static, value. However, rapid imbibition in the pores leads to a breakdown in this assumption and to an 
effective "dynamic" contact angle which depends on velocity. To capture this effect, we use a molecular-kinetics-based wetting model [28-30] to calculate the effective dynamic contact angle

$$
h_{\text {por }} \frac{\partial S}{\partial t}=C_{1} \sinh \left(\sigma C_{2}\left[\cos \left(\theta_{1}^{0}\right)-\cos \left(\theta_{1}\right)\right]\right),
$$

where $C_{1}$ and $C_{2}$ are parameters based on the molecular-kinetic theory [28-30], $\theta_{1}^{0}$ is the equilibrium contact angle, and $\theta_{1}$ is the dynamic contact angle. The left-hand side of (22) is the wetting speed from the literature[28-30] and the right-hand side is the model itself. We follow the approach taken by Reddy et al. [14] for calculation of the $C_{i}$ parameters. This equation is solved for $\cos \left(\theta_{1}\right)$ and used in (21) to calculate the capillary pressure.

Combining the governing equation (16) with the model for pressure gradient (19), along with the calculated values for the permeability, gas pressure (20), and capillary pressure (21) yields the complete model for closed, cylindrical features,

$$
-h_{\mathrm{por}} \phi \frac{\partial S}{\partial t}=\frac{1}{\mu} \frac{\phi r^{2}}{8}\left(\frac{\frac{p_{\mathrm{gas}}^{0} \bar{n}}{1-S}-\frac{2 \sigma \cos \left(\theta_{1}\right)}{r}-p_{\mathrm{lub}}}{S h_{\mathrm{por}}}\right) .
$$

This model for the pore saturation is coupled to the lubrication flow in two ways. First, the lubrication pressure directly appears in (23), influencing the saturation. Secondly, the velocity of liquid flowing into the porous medium (17) is included as the upper-wall velocity, $\boldsymbol{B}_{1, \mathrm{n}}$ in (1), ensuring that conservation of mass is enforced. Of course, if this expression is being evaluated in a multiphase flow simulation, including both liquid and gas phases in the lubrication layer, we can simply set $\partial S / \partial t=0$ in gas regions, as liquid can flow neither into nor out of the pores. Similarly, if $S$ approaches 1, which would happen as all gas leaves the pores $(\bar{n} \rightarrow 0)$, the capillary interface would disappear, leading to the removal of the pressure gradient in the pore, $d p_{\text {por }} / d z=0$.

\subsubsection{Closed-feature gas diffusion}

As mentioned in $\S 2.2 .1$, gas is trapped when liquid fills closed pores. This gas compresses, leading to high gas pressures and accelerating gas diffusion. While rigorous understanding of this behavior would require a full accounting of gas inventory in the pores and the lubrication layer, we choose to take a simpler approach. If we assume that there is much more liquid 
in the lubrication layer then there is in the pores, we can treat the lubrication layer as an infinite sink of dissolved gas. This leaves only the liquid in the pore to model.

The gas flux in the pore can be written $J=-D(\partial C / \partial z)$, where $D$ is the coefficient of diffusion for gas in liquid, and $C$ is the concentration. Assuming a linear concentration profile through the pore, the flux can be re-written using a finite difference,

$$
J=-D \frac{\partial C}{\partial z}=-D \frac{\left.C\right|_{z=h_{1}+S h_{\mathrm{por}}}-\left.C\right|_{z=h_{1}}}{S h_{\mathrm{por}}} .
$$

The concentration at the liquid-gas interface $\left(z=h_{1}+S h_{\text {por }}\right)$ can be estimated using Henry's law, which relates the concentration to the gas pressure, $p_{\text {gas }}=\left.k_{H} C\right|_{z=h_{1}+S h_{\mathrm{por}}}$, where $k_{H}$ is Henry's law constant for the gas. If the liquid were exposed to air prior to the processing, equilibrium of the gas to atmospheric pressure can also be assumed as the condition at the open end of the pore, $p_{\mathrm{atm}}=\left.k_{H} C\right|_{z=h_{1}}$. This allows (24) to be written as

$$
J=-\frac{D}{S h_{\mathrm{por}} k_{H}}\left(p_{\text {gas }}-p_{\text {atm }}\right)=-\frac{D P_{\text {gas }}^{0}}{S h_{\text {por }} k_{H}}\left(\frac{\bar{n}}{1-S}-1\right)
$$

after substituting (20) for $p_{\text {gas }}$ and assuming that $P_{\text {gas }}^{0}=p_{\text {atm }}$.

A mass-conservation equation can be derived to determine the remaining gas inventory. Equating the rate of mass loss with the flux in a single pore yields

$$
\frac{\partial \bar{n}}{\partial t}=\pi r^{2} J
$$

If we scale this equation to the continuum level using the porosity and then substitute in (25). After some algebraic manipulation and simplification, (26) can be rewritten

$$
\frac{\partial \bar{n}}{\partial t}=-\left(\frac{R T D}{h_{\mathrm{por}}^{2} k_{H}}\right) \frac{1}{S}\left(\frac{\bar{n}}{1-S}-1\right) .
$$

Here, $R$ is the universal gas constant and $T$ is the temperature. Equation (27) can be solved simultaneously with (23) to account for the diffusion of gas out of the pores.

\subsubsection{Open-feature flow}

In contrast to the closed-feature flow discussed in $\$ 2.2 .1$, a connected, fully-porous medium allows flow in the transverse direction in addition to the normal direction. This material may still be structured, as illustrated in figure 3. Conversely from figure 2, figure 3 


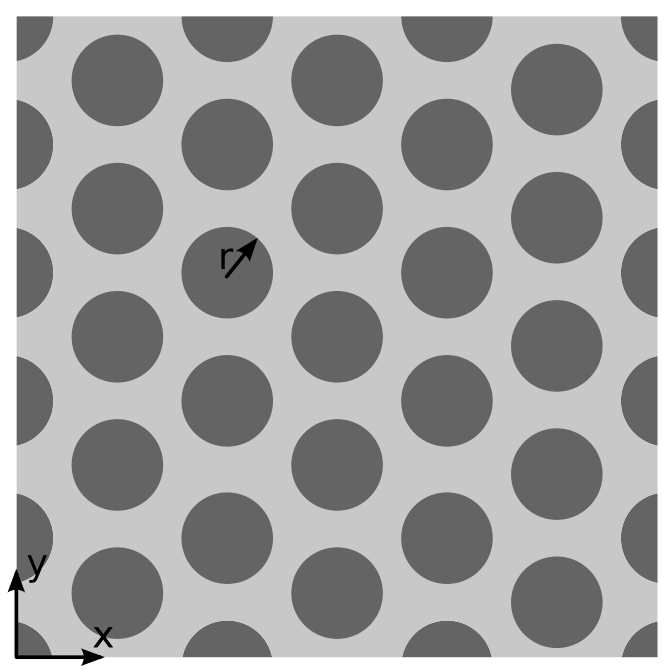

Figure 3: Sample illustration of an open-feature porous material, shown in the $x-y$ plane, featuring an array of hexagonal-packed solid pillars in an otherwise open material. Light gray regions are pores while the dark gray is impermeable material.

consists of solid pillars surrounded by open regions that allow transverse fluid flow. As before, the cylinders have a radius $r$ and thickness $h_{\text {por }}$. The open regions are fluid-mechanically connected to the lubrication layer at $z=h_{1}$ but are not open at the top, $z=h_{3}$.

For open features, all terms in (15) are necessary. However, as in $§ 2.2 .1$, we still need a model for the last term in (15), which represents flow in the normal direction from the neighboring lubrication region. Derivation of the pressure gradient in this term is not as straight-forward as it is for the closed features of $\S 2.2 .1$, as the geometry is more complex. However, one can imagine a form similar to (19). For open features, we assume that the gas can move throughout the porous medium freely (due to $\mu_{\text {gas }} \ll \mu_{\text {liq }}$ ) and that it does not pressurize as it does in the closed feature case; this leads to $p_{\text {gas }}=p_{\text {atm }}$. This holds for the case when the features are not fully saturated, $S<1$. If the features are saturated, $S=1$, unlike the closed-feature case, liquid can still flow in or out of the features, depending on the pore pressure, $p_{\text {por }}$, and the local lubrication pressure in the adjacent layer. Therefore, the pressure gradient has a different form when the features are fully saturated,

$$
\left.\nabla_{\mathrm{n}} p_{\mathrm{por}}\right|_{z=h_{1}}=\left\{\begin{array}{ll}
\frac{p_{\mathrm{atm}}-\frac{2 \sigma \cos \left(\theta_{1}\right)}{r_{\mathrm{por}}}-p_{\mathrm{lub}}}{S h_{\mathrm{por}}} & S<1 \\
\frac{p_{\mathrm{por}}-p_{\mathrm{lub}}}{2 h_{\mathrm{por}}} & S=1
\end{array} .\right.
$$

Here, $r_{\text {por }}$ is the average pore radius. 
Values for the permeability can be calculated using a series solution to the flow past an array of cylinders, originally derived by Sangani and Acrivos [31] and later by Drummond and Tahir [32]. The geometry described in figure 3 is orthotropic, with a different value for the permeability in the $z$ direction as compared to the transverse direction. Following a similar methodology to that presented in $\S 2.2 .1$, but using the drag force solution of Drummond and Tahir [32], we arrive with the permeability tensor

$$
\boldsymbol{K}=\left[\begin{array}{ccc}
K_{x x} & 0 & 0 \\
0 & K_{x x} & 0 \\
0 & 0 & 2 K_{x x}
\end{array}\right]
$$

Here, the permeability expression $K_{x x}$ is the series solution [32]

$$
K_{x x}=\frac{\pi r^{2}}{8 \epsilon}\left[\ln \left(\frac{1}{\epsilon}\right)-1.353663936+2 \epsilon-\frac{\epsilon^{2}}{2}-\frac{0.358221 \epsilon^{3}}{1+2.26579 \epsilon^{3}}\right],
$$

where $\epsilon=1-\phi$ is the feature density. This form assumes that $z$ is the normal direction, although this tensor could easily be rotated for different orientations.

In $§ 2.2 .1$, the saturation $S$ is treated as the independent variable, as the pore pressure $p_{\text {por }}$ did not appear in the final expression (23). In (15), however, both $S$ and $p_{\text {por }}$ appear as unknowns. To account for the additional unknown we follow the methodology presented by Cairncross et al. [33] to derive a relationship between capillary pressure and saturation. First, we recognize that the liquid pressure in the pores, $p_{\text {por }}$, is related to the gas pressure, $p_{\text {gas }}$, by the capillary pressure, $p_{\text {cap }}=p_{\text {gas }}-p_{\text {por }}$. We then use the chain rule to rewrite the time derivative in the first term of (15) as

$$
\frac{\partial S}{\partial t}=\left(\frac{\partial S}{\partial p_{\text {cap }}}\right)\left(\frac{\partial p_{\text {cap }}}{\partial p_{\text {por }}}\right)\left(\frac{\partial p_{\text {por }}}{\partial t}\right)=-\left(\frac{\partial S}{\partial p_{\text {cap }}}\right)\left(\frac{\partial p_{\text {por }}}{\partial t}\right) .
$$

This reorganization leaves $p_{\text {por }}$ as the independent variable, assuming that this relationship between $S$ and $p_{\text {por }}$ is known [33]. These relationships can be analytically or experimentally obtained, and often exhibit hysteresis. Here, however, we derive a simple analytical form following the method presented by Cairncross et al. [33].

In a hexagonal-packed cylinder configuration, the cylinder radius $r$ and porosity $\phi$ characterize fully the microstructure. For a given arrangement the minimum pore distance, $r_{\text {por }}^{\min }$, 
is given by

$$
r_{\mathrm{por}}^{\min }=-2 r+\frac{\sqrt{2 \pi} r^{2}}{3^{1 / 4} \sqrt{r^{2}(1-\phi)}},
$$

while the maximum pore spacing, $r_{\text {por }}^{\max }$, is

$$
r_{\text {por }}^{\max }=2 r(\sqrt{3}-1)+\sqrt{3} r_{\text {por }}^{\min } .
$$

If we assume that the pore size distribution is Gaussian, with a mean value of $r_{\mathrm{por}}^{\operatorname{mean}}=\left(r_{\mathrm{por}}^{\max }+\right.$ $\left.r_{\text {por }}^{\min }\right) / 2$ and a standard deviation of $r_{\text {por }}^{\text {stdv }}=\left(r_{\text {por }}^{\text {mean }}-r_{\text {por }}^{\min }\right) / 2$, the probability distribution function of the pore size distribution is

$$
F\left(r_{\text {por }}\right)=\frac{1}{\sqrt{2 \pi} r_{\text {por }}^{\text {stdv }}} \exp \left[\frac{-\left(r_{\text {por }}-r_{\text {por }}^{\text {mean }}\right)^{2}}{2 r_{\text {por }}^{\text {stdv }} r_{\text {por }}^{\text {stdv }}}\right] .
$$

Further following Cairncross et al. [33], the saturation as a function of pore size is

$$
S\left(r_{\text {por }}\right)=\frac{\int_{0}^{r_{\text {por }}} r^{2} F\left(r_{\text {por }}\right) d r}{\int_{0}^{\infty} r^{2} F\left(r_{\text {por }}\right) d r} .
$$

Assuming the Young-Laplace form of the capillary radius, $r_{\text {por }}=2 \sigma \cos \left(\theta_{1}\right) / p_{\text {por }}$, along with (35) yields a functional form for the capillary pressure-saturation relationship, $S\left(p_{\text {por }}\right)$. The relative permeability could be calculated using similar techniques [33]. This expression allows calculation of $\partial S / \partial p_{\text {por }}$, completing the description of the governing equation for open-feature flow,

$$
h_{\text {por }} \phi \frac{\partial S}{\partial p_{\text {por }}} \frac{\partial p_{\text {por }}}{\partial t}=-\frac{h_{\text {por }} k_{\text {rel }} K_{x x}}{\mu} \nabla_{\|}^{2} p_{\text {por }}+\left.\frac{2 k_{\text {rel }} K_{x x}}{\mu} \nabla_{\mathrm{n}} p_{\text {por }}\right|_{z=h_{1}} .
$$

Of course, (29-35) were derived specifically for the assumed geometry of figure 3 . When full saturation $(S=1)$ is reached, this equation is replaced by $\frac{\partial S}{\partial t}=0$. However, (36) is general, and the chosen model for $K_{x x}$ and $\left.\nabla_{\mathrm{n}} p_{\mathrm{por}}\right|_{z=h_{1}}$ may be chosen specifically for any other pore configuration.

\section{Numerical method}

The multiphase lubrication and porous flow equations of $\S 2$ are implemented into the Galerkin finite element method code Goma [34]. We chose isoparametric quadrilateral shell elements with bi-linear (Q1) Lagrange interpolation functions, the implementation of which was discussed in a previous work [11]. The mesh sizes are chosen to balance resolution of the 
physics with computational speed, but also meets the requirement that a single element must be significantly larger than the feature size. A more refined mesh allows better resolution of spatially varying macroscopic properties. The nonlinear residual equations are solved using a fully-coupled Newton-Rhapson method, and the resulting linear equations are solved with direct (LU) and iterative (GMRES) algorithms deployed from the third-party library Trilinos [35]. Transient problems are advanced in time with the backward Euler method.

As mentioned in $\S 2$, physical properties and system characteristics, such as material thicknesses or pore properties, may vary spatially. In order to handle complex shapes and distributions, such as those that may arise in nano-manufacturing, a pattern-to-mesh algorithm was developed and implemented into Goma. This algorithm enables a CAD file representing the spatial variations in the pore design to be mapped onto the computational mesh and used for simulations. First, the CAD description is exported as a gray-scale raster image representing spatial variations in a given property. Separate exports may be done to get different spatial dependencies for different porous properties. Next, image editing software may be used to simplify or smooth the design in a way that is appropriate for the overall quality of the computational mesh that is to be used. The image is then exported as a gray-scale image over the spatial coordinates, from which the porous-medium properties are scaled. Finally, the text file is read into Goma and mapped onto the mesh.

Mapping of image pixel values onto an unstructured mesh requires a least-squares fit of the pixel values using the finite element basis functions. The finite element representation of the pixel field is expressed as

$$
p^{h}(\boldsymbol{x})=\sum_{i=1}^{N} p_{i} \phi_{i}(\boldsymbol{x})
$$

where $p^{h}$ is the finite element representation of the pixel field, $p_{i}$ are the nodal values of the pixel field, and $\phi_{i}(\boldsymbol{x})$ are the finite element basis functions, with $i$ representing a node out of $N$ total nodes on the mesh. With this representation, the least-squares fit is represented by the following system of algebraic equations:

$$
p_{j}^{*}-p^{h}\left(\boldsymbol{x}_{j}\right)=p_{j}^{*}-\sum_{i=1}^{N} p_{i} \phi_{i}\left(\boldsymbol{x}_{j}\right)=0 \quad \forall j=1, \ldots, M .
$$

Here, $p_{j}^{*}$ is the value at pixel $j$ of $M$ total pixels, and $\boldsymbol{x}_{j}$ is the associated pixel position. 
Completing the mapping requires solving (38), which is a non-square, $M \times N$ linear system of equations, $\boldsymbol{p}^{*}=\boldsymbol{A} \boldsymbol{p}$, where the matrix is $\boldsymbol{A}=\sum_{i=1}^{N} \phi_{i}\left(\boldsymbol{x}_{j}\right)$. This is most efficiently done by first creating the normal equation by pre-multiplying by the matrix transpose,

$$
\boldsymbol{A}^{\mathrm{T}} \boldsymbol{p}^{*}=\boldsymbol{A}^{\mathrm{T}} \boldsymbol{A} \boldsymbol{p} .
$$

This system of equations is now $M \times M$ and can be solved using standard methods. In Goma, we use a bi-conjugate gradient iterative scheme with a Neumann pre-conditioner, deployed from TriLinos [35].

\section{Numerical demonstrations}

A number of demonstration problems are presented in this section. First, we investigate the time scales for the filling of closed-pores in $\S 4.1$. We then address the problem of filling closed features by squeezing a drop with a porous template $§ 4.2$. The open-pore model is then demonstrated with two problems. First, the problem of a confined drop being absorbed into an open-pore material is examined $\S 4.3$. Then, a drop is allowed to roll down a curved, open-pore surface under the force of gravity while its inventory of liquid is absorbed into the surface ( $\$ 4.4)$. Finally, filling of closed pores with spatially varying properties under multiple squeezing drops is presented in $§ 4.5$. The parameter values used in these demonstration problems were chosen to be relevant for nano-manufacturing problems of interest to the authors and to illustrate key phenomena. For each of these demonstration problems, we have chosen a relative permeability of unity to simplify the analysis. While this choice is questionable, we do not believe that including a model for relative permeability will qualitatively change our results.

\subsection{Flooded closed-feature filling}

In this example, we consider a closed-feature porous region coupled to a lubrication region flooded with liquid, i.e. the lubrication region does not contain a liquid-gas interface. The porous medium consists of cylindrical pores of $h_{\text {por }}=1 \mu \mathrm{m}$ and $r_{\text {por }}=100 \mathrm{~nm}$ with a uniform, constant porosity of $\phi=0.1$. The liquid is taken as water and the gas as air, both initially at atmospheric pressure and room temperature. The lubrication layer is held at a constant 


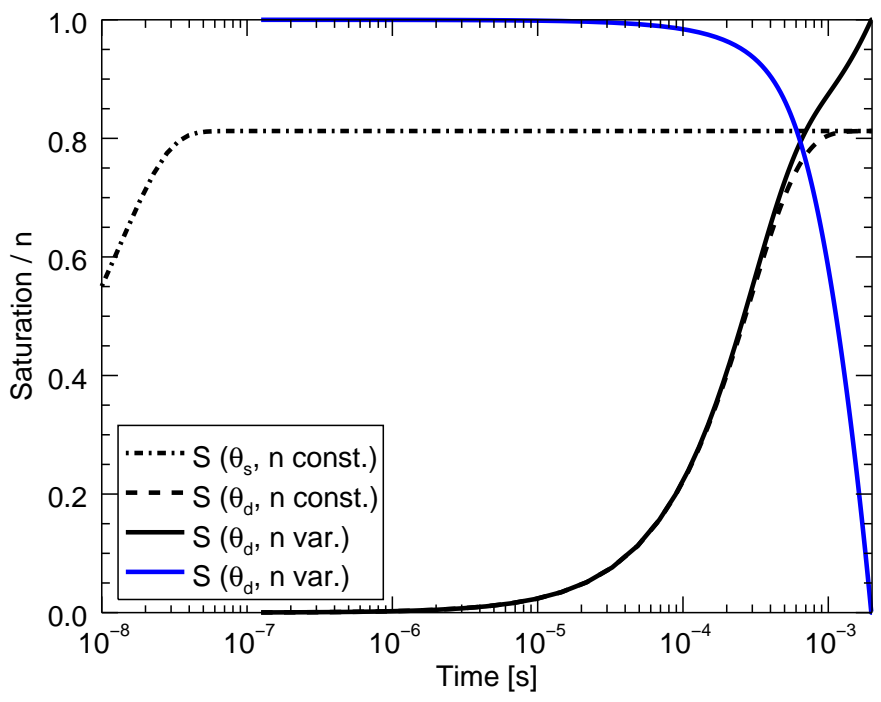

Figure 4: Results from filling of closed features with a flooded lubrication region. The dash-dot curve $(-\cdot-)$ is the saturation predicted with a static contact angle when no gas transport is allowed, the dashed curve (- - ) with a dynamic contact angle, but still no gas transport, and the solid curves are for a dynamic contact angle and allow gas diffusion. Black curves are saturation and the blue curve is the molar gas ratio, $\bar{n}$.

thickness of $h_{\text {lub }}=15 \mu \mathrm{m}$. A static contact angle of $\theta_{1}=30^{\circ}$ is taken between the water and the porous medium structure and the liquid-air surface tension is $\sigma=25$ dyne $/ \mathrm{cm}$. The simulation domain is $50 \mu \mathrm{m}$ square. Simulations are performed using both static and dynamic contact angles (22) both with and without gas diffusion through the pores (§2.2.2). When gas diffusion is included, the diffusivity of air in water is $D=10^{6} \mathrm{~cm}^{2} / \mathrm{s}$, the temperature $T=25^{\circ} \mathrm{C}$, and the Henry's law constant $k_{H}=10^{12} \mathrm{~cm}^{3}$ Barye $/$ mol. A mesh refinement study was not performed due to the one-dimensional nature of this problem. Results of simulations comparing the effect of the contact angle and gas diffusion are shown in figure 4 .

We compare first the effect of contact angle, shown in the dash-dot and dashed curves in figure 4. With a fixed, static contact angle, the features fill to their equilibrium value of $S=0.81$ in less than $0.1 \mu \mathrm{s}$. Such a rapid filling event would require a penetration velocity of nearly $10 \mathrm{~m} / \mathrm{s}$, a significantly higher velocity than is capable of maintaining a static contact angle, even in the small pore space. More physically, the filling curve using the dynamic contact angle model (22) is shown by the dashed line in figure 4 . In this case, the filling happens over $\sim 1 \mathrm{~ms}$, still reaching the same equilibrium saturation value. 
In these first two simulations, the saturation reached an equilibrium value due to the trapping of gas in the pores. The equilibrium value of $S=0.81$ represents a balance between the increased pressure of the gas due to compression (20) and the capillary forces (21). This increased gas pressure, however, increases the equilibrium concentration of gas in the liquid. When (27) is applied, the gas fully dissolves into the liquid and diffuses out of the pores, allowing complete saturation, as shown by the solid curves in figure 4 . The rate of gas dissolution only increases as the pores fill and the pressure increases, leading to gas diffusion on a slower time scale than the initial filling operation, with complete saturation being reached at $2 \mathrm{~ms}$. The relationship between the time scales of the filling process and the gas dissolution process are controlled by the dynamic wetting behavior and gas diffusivity, respectively. This example problem shows that both dynamic wetting behavior and gas dissolution are essential to a predictive model of the process as they lead to experimentally observed filling times.

\subsection{Multiphase closed-feature filling}

Building on the previous result, we now consider the adjacent lubrication layer to initially be partially filled with liquid. As was suggested by the results of the previous section, both dynamic wetting behavior and gas diffusion are included in this simulation. The porous phase consists of uniform, closed pores with height $h_{\text {por }}=1 \mu \mathrm{m}$, radius $r_{\text {por }}=1 \mu \mathrm{m}$, and porosity $\phi=0.05$. The fluids are taken as water and air, with properties identical to those in §4.1. The domain in this simulation is $1 \mathrm{~mm} \times 1 \mathrm{~mm}$, discretized with a $50 \times 50$ element mesh, with an initial fluid thickness of $19.1 \mu \mathrm{m}$. A liquid drop with radius $144 \mu \mathrm{m}$ is centered in the middle of the domain. This simulation is performed in two steps. First, the porous medium is pressed into the liquid drop with a velocity of $5.3 \mathrm{~mm} / \mathrm{s}$. After $3.28 \mathrm{~ms}$ of squeezing, with a gap thickness of $1.73 \mu \mathrm{m}$, the downward motion is stopped and the lubrication layer is held at a constant gap for the remainder of the simulation. A mesh refinement study (not shown here) indicated our chosen mesh resolution provided reasonable accuracy for this demonstration, and the model showed convergence with increased mesh resolution.

Four snapshots from this simulation, as viewed from above though the porous layer, are shown in figure 5 . The first three snapshots (figures $5 \mathrm{a}-5 \mathrm{c}$ ) show that as the drop is spreading 
(a)

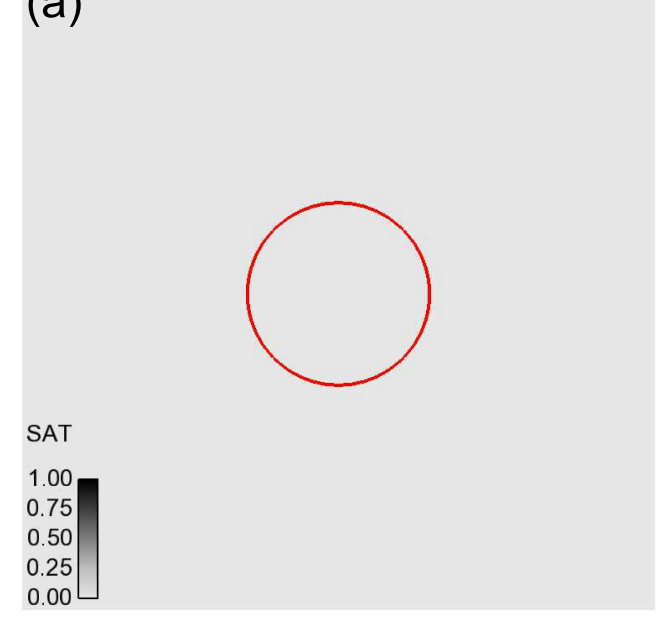

\section{(c)}

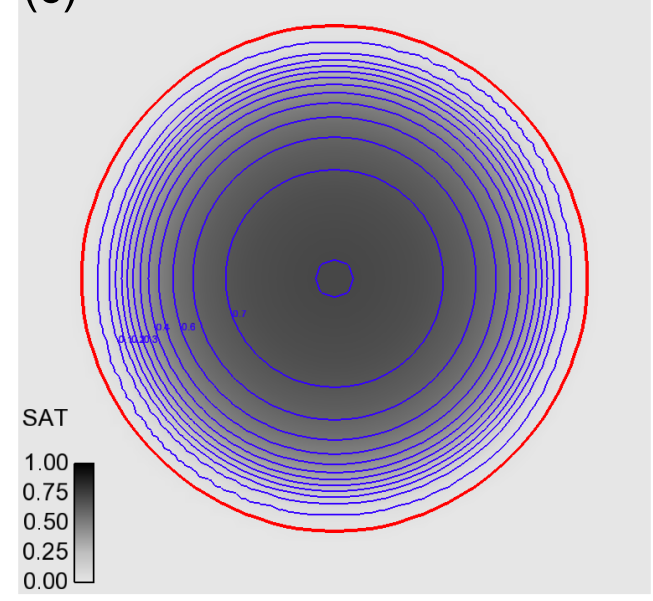

(b)
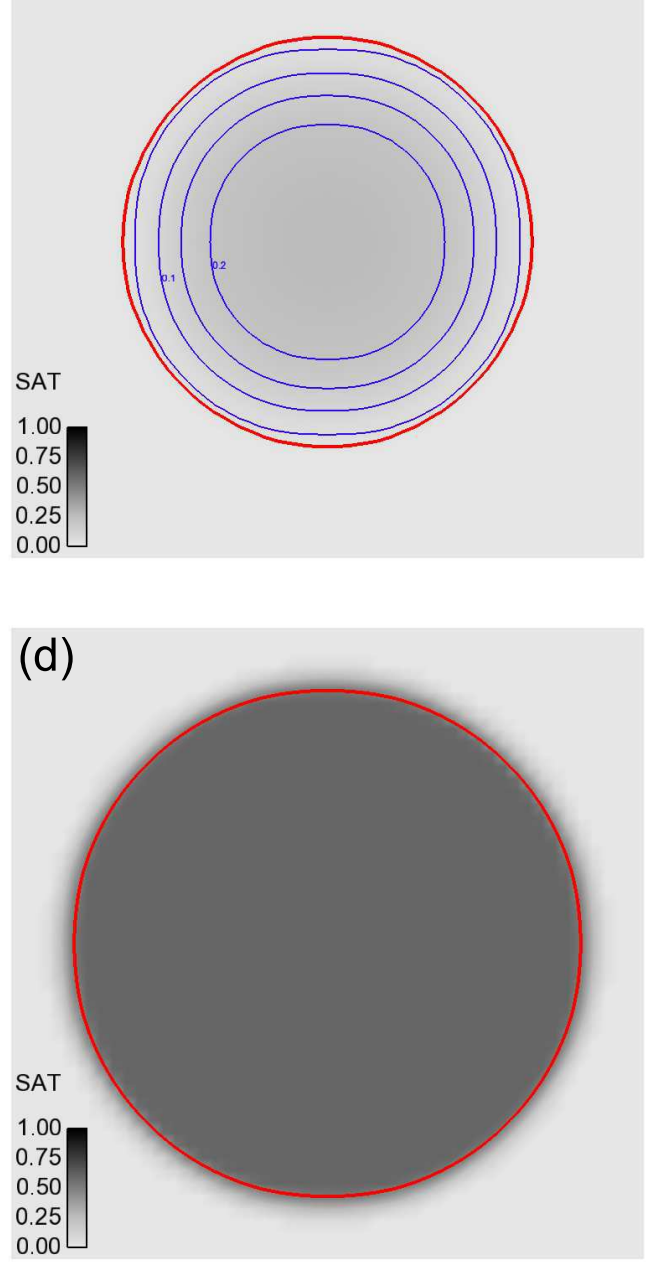

Figure 5: Snapshots of a of closed-feature drop simulation under a uniform template of features at four different simulation times. The red curve represents the location of the liquid-air lubrication interface, while the grayscale coloration and blue contour curves represent the saturation. a) $t=0 \mathrm{~s}, \mathrm{~b}) t=0.003 \mathrm{~s}, \mathrm{c}$ ) $t=0.00323 \mathrm{~s}, \mathrm{~d}) t=2.0 \mathrm{~s}$. 
(a)

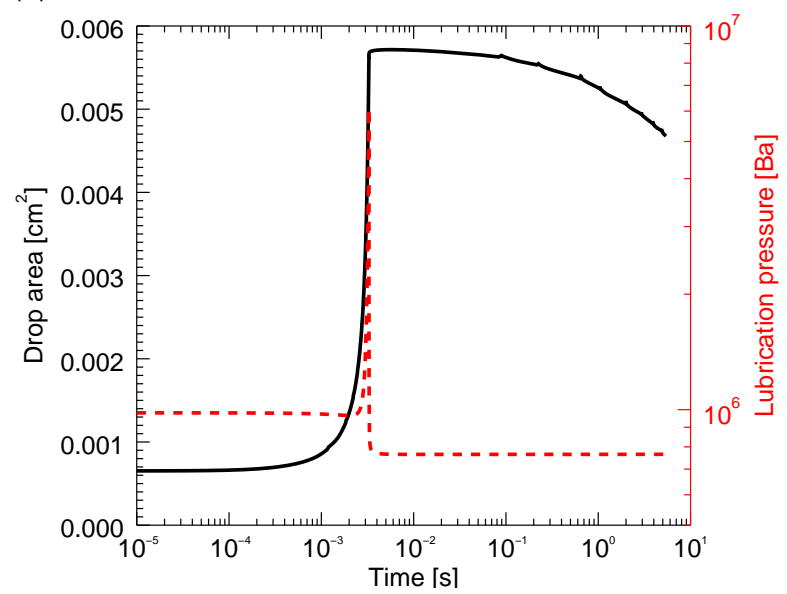

(b)

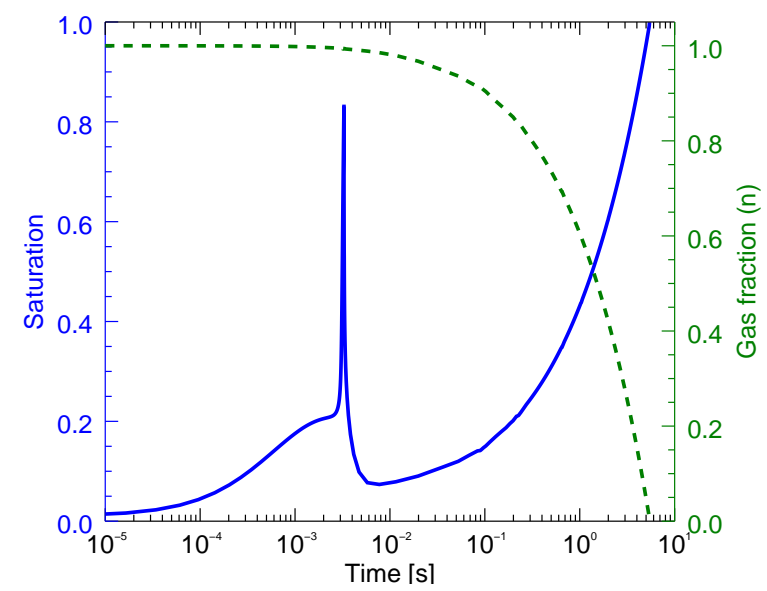

Figure 6: Model predictions of drop area and lubrication pressure (left) and saturation and gas fraction (right) for a closed-feature template. Conditions are given in $\S 4.2$. The drop was squeezed with velocity $0.53 \mathrm{~cm} / \mathrm{s}$ for the first $0.00328 \mathrm{~s}$, then held at a constant lubrication gap thickness. Data taken at the center of the drop. In (a), the area of the liquid drop is shown with the solid black curve and the lubrication pressure is shown by the dashed red curve. In (b), the saturation of the porous media is shown with the solid blue curve while the gas mass fraction is shown by the dashed green curve.

under the downward pressure, the porous layer increases in saturation non-uniformly. The center of the drop shows a higher saturation, due to its longer contact with the liquid in the lubrication layer and the higher pressures generated by the squeezing motion. figure $5 \mathrm{c}$ shows the moment where the squeezing motion is stopped, while figure 5d takes place long after the motion has stopped. figure $5 \mathrm{~d}$ shows that the porous region that is in contact with the drop is uniformly saturated with nearly complete filling of the pores, while the surrounding porous regions are completely unsaturated.

A detailed time history of this simulation is shown in figure 6. During the squeezing phase of the simulation, the saturation of the porous phase grows monotonically, as does the radius of the drop itself. Very little change is seen in the fraction of gas remaining in the pores, as gas dissolution and diffusion occurs at much longer time scales than the saturation of the pores.

As the lubrication pressure begins to spike, the squeezing motion is stopped. This causes a number of significant effects. First the pressure in the liquid layer drops, quickly reaching a level significantly lower than during the squeezing process. The precipitous drop in pressure 
results from the capillary forces in the drop interface and the cessation of viscous forces. In turn, the pressurized gas trapped in the pores quickly pushes liquid out of the pores, reducing the overall saturation level.

Following the rapid pressure change after squeezing, a slower transient behavior begins. Because of the capillary suction effect of the pores, liquid continues to invade the media. Gas also diffuses out of the pores at these slower time scales, allowing the pores to become fully saturated. The loss of liquid from the drop into the pores is reflected in the shrinking of the drop radius, shown in figure $6 \mathrm{a}$.

\subsection{Multiphase open-feature filling}

We now consider a open pore structure, as discussed in $§ 2.2 .3$. Here, a liquid drop of radius $10 \mathrm{~mm}$ and height $5 \mathrm{~mm}$ is placed under a open-feature porous layer. This porous layer is modeled as a square-packed array of cylinders (30) with cylinder radius of $100 \mu \mathrm{m}$, height $500 \mu \mathrm{m}$, and porosity $\phi=0.8$. The simulation domain is $50 \mathrm{~mm} \times 50 \mathrm{~mm}$, discretized with a $50 \times 50$ mesh.

In this simulation, both bounding layers (substrate and porous layers) are held fixed in place. Where the liquid contacts the porous layer, the liquid is sucked into the porous layer, subsequently shrinking the size of the drop, as shown in the snapshots of figure 7 . However, unlike in the previous simulations in which the features where closed and unable to communicate in the transverse direction, this porous medium is able to transmit liquid laterally, effectively pumping liquid out of the drop and distributing it through the porous layer. Details of this simulation are shown in figure 8. The saturation at the center of the drop increases steadily as the drop shrinks in area. However, the saturation levels off as the drop disappears and the liquid distributes evenly through the porous layer, which happens shortly after $1 \mathrm{~ms}$. Over the next ten seconds, the saturation decreases uniformly to near its initial level.

\subsection{Multiphase open-feature filling on a curved surface}

In this example we demonstrate the generalized shell element formulation by deploying the model in a three-dimensional geometry. Specifically, we consider a confined liquid drop absorbing into a curved porous layer while flowing due to gravity. The substrate is a 
(a)
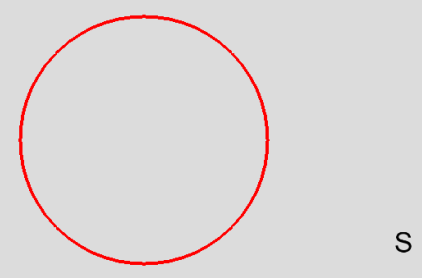

0.125

0.117

0.110

0.102

0.095

(c)

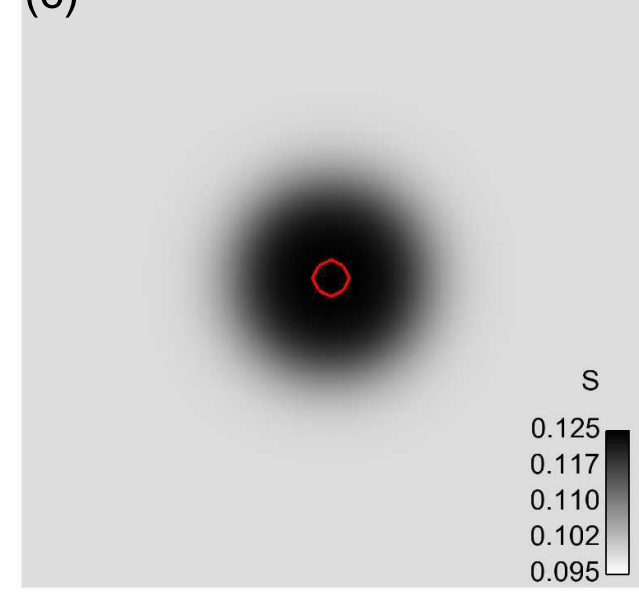

(b)

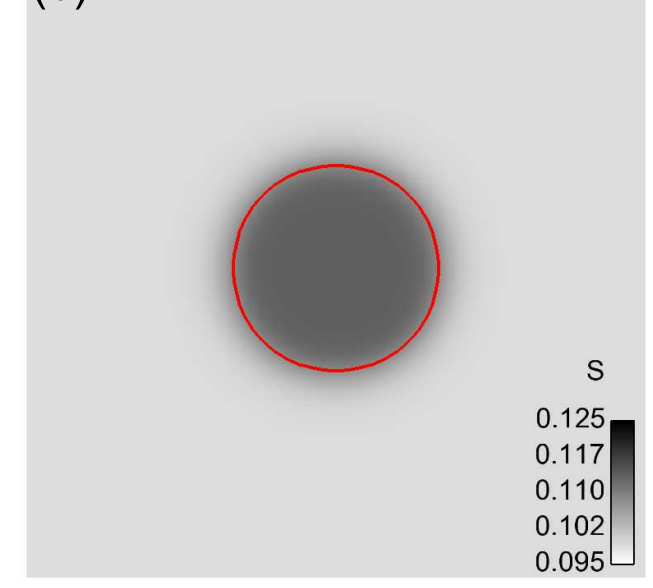

(d)

$\mathrm{S}$

0.125

0.117

0.110

0.102

0.095

Figure 7: Results of open-feature drop simulations under a uniform template of features at four different simulation times. The red curve represents the location of the liquid-air lubrication interface, while the grayscale coloration represents the saturation. (a) $t=10^{-6} \mathrm{~s}$, (b) $t=10^{-3} \mathrm{~s}$, (c) $t=1.95 \times 10^{-3} \mathrm{~s}$, (d) $t=67 \mathrm{~s}$. 


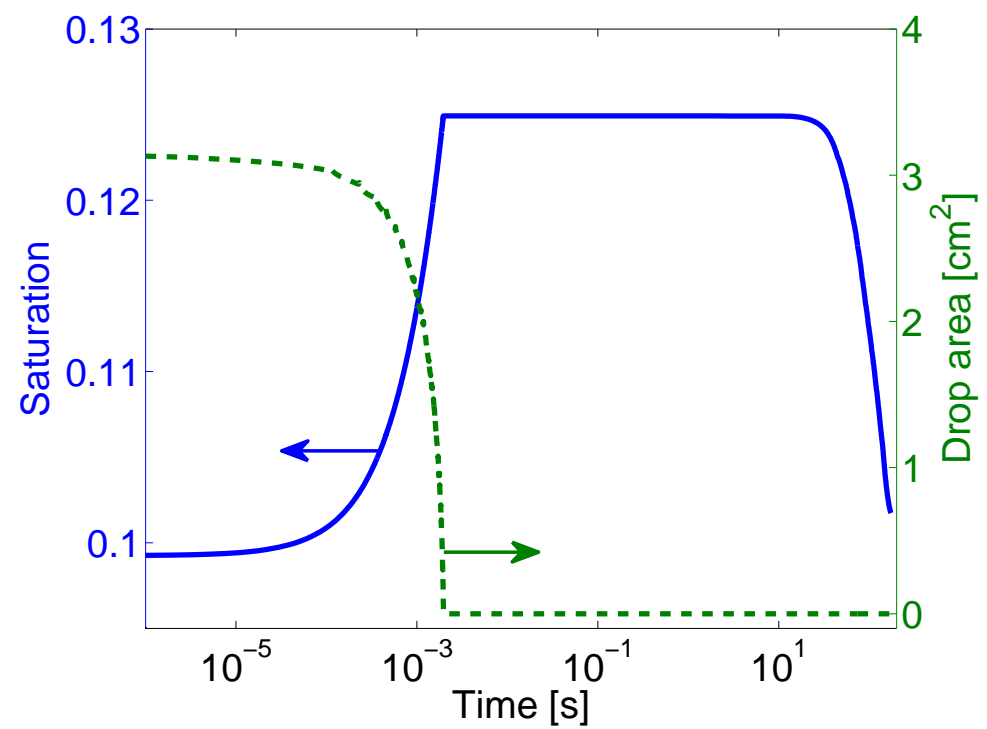

Figure 8: Saturation (measured at the drop center) and drop area for an open-feature drop simulation. Saturation is the solid curve and drop area the dashed green curve.

horizontally-oriented cylinder with radius $5 \mathrm{~cm}$ and length $2 \mathrm{~cm}$. The exterior of this cylinder is an open-feature porous material with porosity $\phi=0.1$, pore radius $0.1 \mathrm{~mm}$ and pore height $2.5 \mathrm{~mm}$. Adjacent to this porous surface is another rigid cylinder of slightly larger radius, leaving a $0.3 \mathrm{~cm}$ gap between the porous surface and the outer cylinder. In the gap is a drop of water of radius $0.3 \mathrm{~cm}$, centered horizontally but at a 12 degree angle off of the vertical.

Eight snapshots from this simulation are shown in figure 9. In these images, the color represents the degree of saturation of the underlying porous layer, and the location of the liquid drop edges are shown with the dark green curve. Only a section of the cylinder is shown in this figure. figure 9a shows the initial condition, where the porous layer is initialized to a saturation of $S=0.1$ for numerical ease.

A competition between gap-lubrication transport under gravity and open-porous flow is observed in the next four frames (figures 9b-9e), where two competing physics can be seen. First, the drop is rolling down the curved surface under the force of gravity. At the same time, the liquid within the drop is being absorbed into the underlying porous layer, shrinking the volume of liquid remaining in the drop. Directly under the drop, the porous layer reaches its most saturated levels. However, the open nature of the pores quickly sucks the liquid away from the drop, saturating the areas around the drop. In figure 9f, the porous layer 

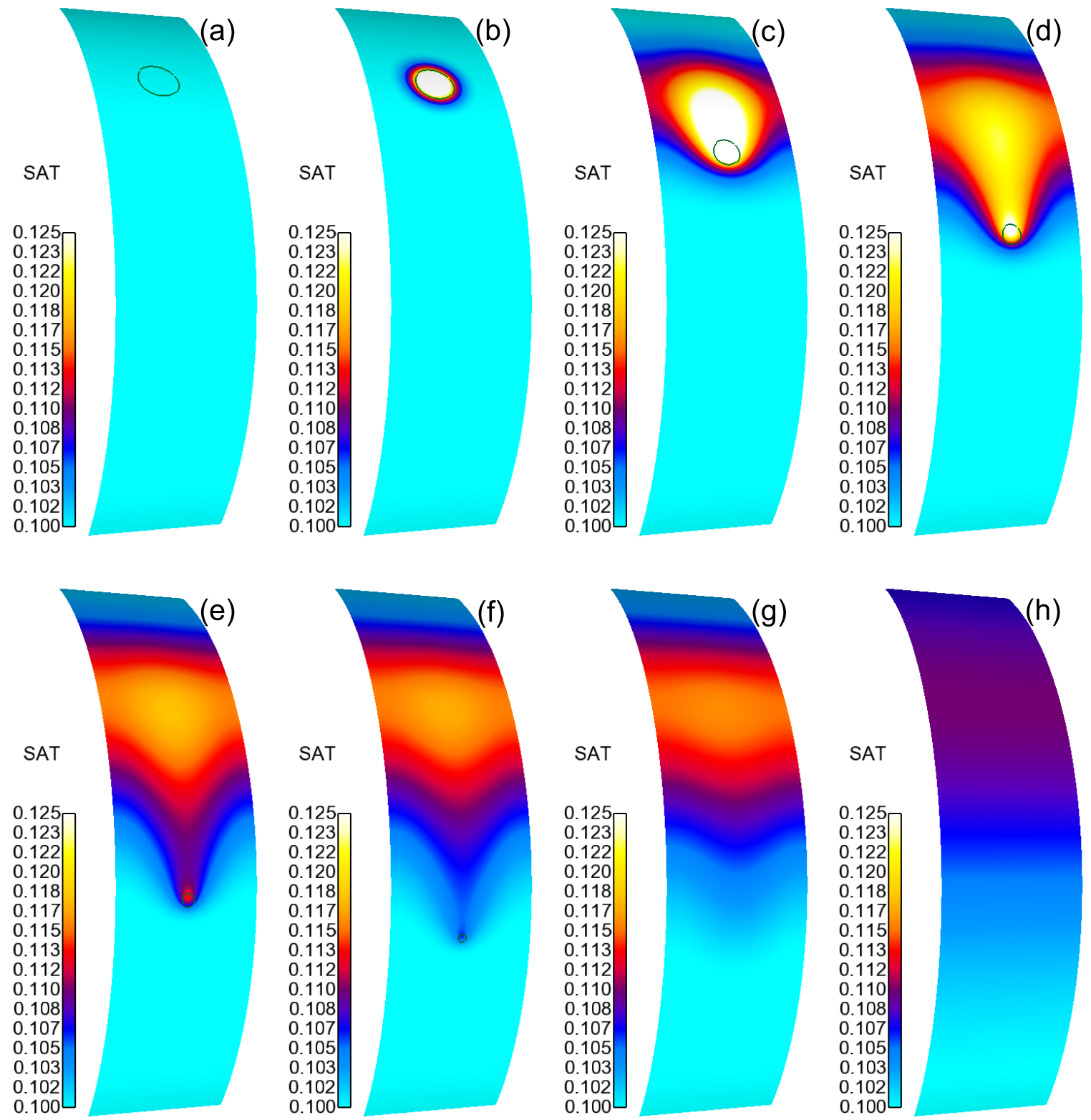

Figure 9: Snapshots from the simulation of a confined liquid drop rolling down a curved, porous (openfeature) surface. The dark green curve represents the location of the liquid-air interface, and the color scale is the saturation of the porous media. (a) $t=0 \mathrm{~s}$, (b) $t=0.001 \mathrm{~s}$, (c) $t=0.010 \mathrm{~s}$, (d) $t=0.013 \mathrm{~s}$, (e) $t=0.020 \mathrm{~s},(\mathrm{f}) t=0.025 \mathrm{~s},(\mathrm{~g}) t=0.030 \mathrm{~s},(\mathrm{~h}) t=0.080 \mathrm{~s}$. 


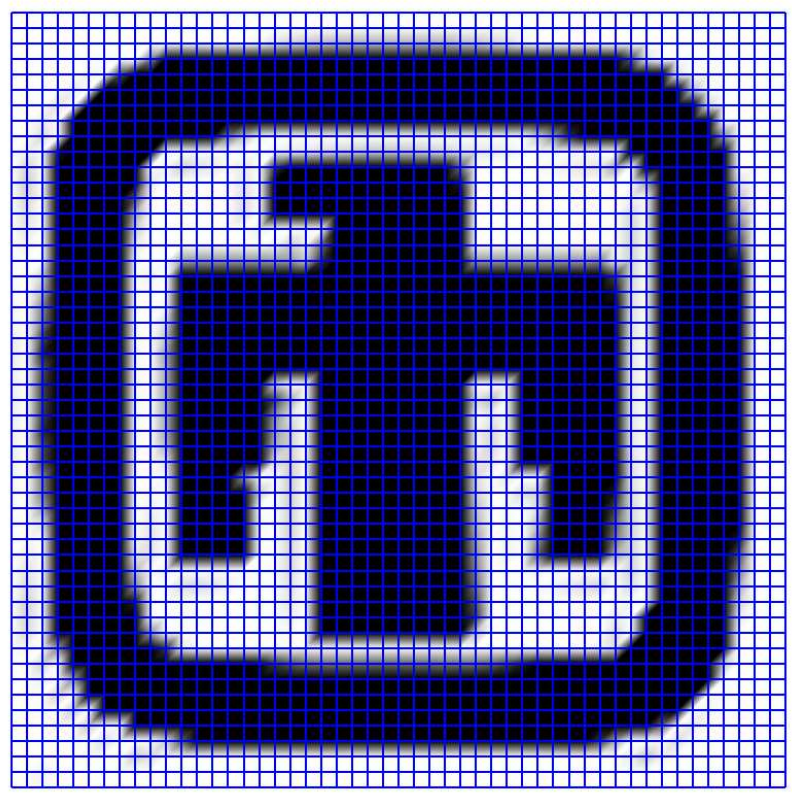

Figure 10: Computational mesh and porosity pattern for simulations of $\S 4.5$. White regions represent unpatterned regions $(\phi=0)$, black is $\phi=0.2$, and there is a gradient of gray-scale regions between. The computational domain is $90 \mu \mathrm{m}$ wide, and the mesh lines are shown in blue.

absorbs the remaining volume of the drop. Then, in the final two frames (figures $9 \mathrm{~g}-9 \mathrm{~h}$ ), the liquid drop is gone, but the porous layer continues to redistribute the liquid until it is nearly uniform in figure $9 \mathrm{~h}$.

\subsection{Patterned, multiphase closed-feature filling}

Finally, we present an example problem that utilizes the pattern-to-mesh capability presented in $\S 3$ to study the flow through a thin porous medium with spatially-varying properties. The drops do not initially cover the entire patterned area. The pattern used in this study, along with the simulation mesh, is shown in figure 10. Within the pattern, the porosity varies $\phi=0-0.2$. Fluid and pore properties are the same as in $\S 4.1$. The lubrication layer initially consists of five liquid drops placed in an equally-spaced array. The initial lubrication height is $5.6 \mu \mathrm{m}$ and the initial drop radius is $15 \mu \mathrm{m}$. The template is held with a no-normal-stress boundary condition and is allowed to be lowered by the capillary forces.

Three snapshots of this simulation are shown in figure 11. Figure 11a shows the state shortly after the initial condition. The liquid drops are near their initial configuration, and the patterned, porous region is just beginning to take up liquid. In figure 11b, the drops 
(a)

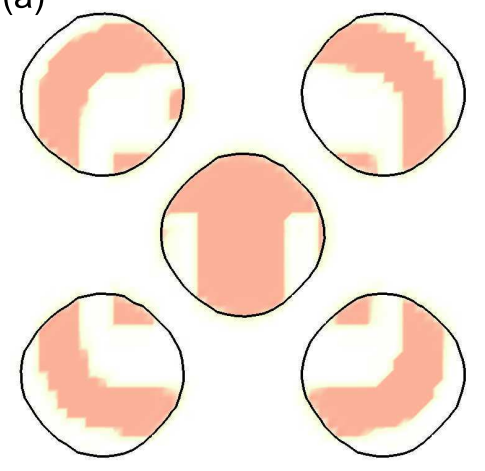

(b)

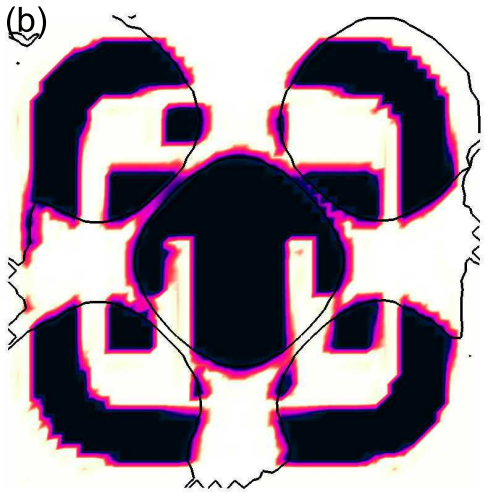

(c)

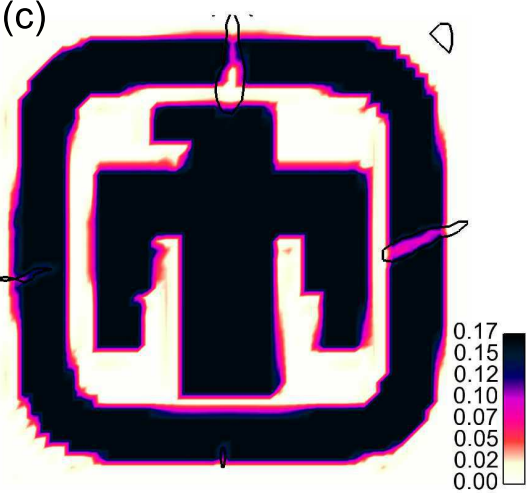

Figure 11: Results of closed-feature simulations under the patterned template shown in figure 10. The dark green curves represent the location of the liquid-air lubrication interface, while the color scheme represents the relative saturation, $S \phi$. (a) $t=10^{-6} \mathrm{~s}$, (b) $t=12 \mathrm{~s}$, (c) $t=920 \mathrm{~s}$.

have spread and nearly coalesce in the middle of the domain, and some of them have spread outside of the domain at the edges. The patterned regions that are in contact with liquid are fully saturated, while the regions outside the drops remain empty. Finally, in figure 11c, the drops have fully merged and covered nearly the entire domain. All patterned regions covered with liquid are fully saturated.

In terms of a practical processing application, it is important that a few air bubbles have been trapped in the lubrication region (figure 11c) and the patterned areas covered by these bubbles remain unsaturated. This highlights the need for this type of simulation, to intelligently design imprinting process to minimize air bubble entrapment and eliminate pattern defects.

\section{Summary and recommendations}

In this paper, we derived a reduced-order model for flow in thin porous media. We implemented this model in a shell finite-element framework. Starting with the generalized Darcy's equations, we reduced the model using thin-region approximations similar to those used for shell mechanics theory and Reynolds' lubrication flow. These equations were first presented in general, and then two specializations were derived. The first specialization addressed structures (holes) not transversely connected such that no lateral transport of fluid occurs. A gas diffusion model in this closed-pore case was also postulated. The second 
specialization addressed interconnected or open-feature pore space.

We demonstrated our models with five example problems mostly related to micro-replication and imprint lithography applications. The first two problems utilized the closed-feature model, demonstrating its use when the porous structure is squeezed onto a liquid drop. Next the open-feature model was used in a similar application. In the fourth example, the power of this model on curved shell elements was demonstrated, as a drop rolls down and is absorbed into a curved porous substrate. Finally, the use of our pattern-to-mesh capability is demonstrated by spatially varying the porous properties to fill a patterned porous medium.

This model can be applied to address the physics and design of manufacturing processes and the performance of absorbent paper products. Because it is a reduced-order two-dimensional model, it is significantly more computationally efficient than a full 3-D counterpart, leading to a significant reduction in model size (element count) and better quality meshes. It is most useful when combined with other reduced-order models for the continuous phase, such as the lubrication equation. A logical extension to pursue would be to couple it with the thin-film equations, allowing the capture of porous suction from free drops or liquid standing on other surfaces. Another significant extension would be to couple these reduced-order equations for flow through porous media with continuum 3-D fluid regions, allowing the study of more complex geometries. Additional 3-D simulations should also be conducted to show the applicability of this model.

\section{Acknowledgments}

The authors would like to thank R. T. Bonnecaze and S. V. Sreenivasan from the University of Texas for a fruitful collaboration and educating us on nanoimprint lithography. We appreciate the work of E. M. Benner analyzing experimental data to inform the construction of the examples shown here. We also appreciate the peer review and discussion of this manuscript with our Sandia colleagues.

Sandia National Laboratories is a multi program laboratory managed and operated by Sandia Corporation, a wholly owned subsidiary of Lockheed Martin Corporation, for the U.S. Department of Energy's National Nuclear Security Administration under contract DEAC04-94AL85000. 
[1] Bear, J.. Dynamics of Fluids in Porous Media. Dover Books on Physics and Chemistry; Dover Publications; 1988. ISBN 9780486656755.

[2] Darcy, H.. The Public Fountains of the City of Dijon. Kendall Hunt Publishing; 1856. English translation by Patricia Bobeck.

[3] Whitaker, S.. Flow in porous media I: A theoretical derivation of Darcy's law. Transport in porous media 1986;1(1):3-25.

[4] Dagan, G.. Models of groundwater flow in statistically homogeneous porous formations. Water Resources Research 1979;15(1):47-63.

[5] Bear, J., Verruijt, A.. Modeling groundwater flow and pollution; vol. 2. Springer; 1987.

[6] Payatakes, A., Tien, C., Turian, R.. A new model for granular porous media: Part I. Model formulation. AIChE Journal 1973;19(1):58-67.

[7] Bathe, K.J., Dvorkin, E.N.. A formulation of general shell elements - the use of mixed interpolation of tensorial components. International Journal for Numerical Methods in Engineering 1986;22(3):697-722. doi:10.1002/nme.1620220312.

[8] Ahmad, S., Irons, B.M., Zienkiewicz, O.C.. Analysis of thick and thin shell structures by curved finite elements. International Journal for Numerical Methods in Engineering 2005;2(3):419-451.

[9] Hori, Y.. Hydrodynamic Lubrication. Springer Tokyo; 2006. doi:10.1007/4-431-27901-6.

[10] Oron, A., Davis, S.H., Bankoff, S.G.. Long-scale evolution of thin liquid films. Reviews of Modern Physics 1997;69(3):931-980. doi:10.1103/RevModPhys.69.931.

[11] Roberts, S.A., Noble, D.R., Benner, E.M., Schunk, P.R.. Multiphase hydrodynamic lubrication flow using a three-dimensional shell finite element model. Computers \& Fluids 2013;87(0):12 - 25. doi:10.1016/j.compfluid.2012.08.009.

[12] Resnick, D.J., Sreenivasan, S., Willson, C.G.. Step \& flash imprint lithography. Materials Today 2005;8(2):34 - 42. doi:10.1016/S1369-7021(05)00700-5. 
[13] Rowland, H.D., Sun, A.C., Schunk, P.R., King, W.P.. Impact of polymer film thickness and cavity size on polymer flow during embossing: toward process design rules for nanoimprint lithography. Journal of Micromechanics and Microengineering 2005;15(12):2414-2425.

[14] Reddy, S., Schunk, P.R., Bonnecaze, R.T.. Dynamics of low capillary number interfaces moving through sharp features. Physics of Fluids 2005;17(12):122104 (pages 6). doi:10.1063/1.2140691.

[15] Reddy, S., Bonnecaze, R.T.. Simulation of fluid flow in the step and flash imprint lithography process. Microelectronic Engineering 2005;82(1):60 - 70. doi:10.1016/j.mee.2005.06.002.

[16] Reddy, S.. Fluid and solid mechanics in the step and flash imprint lithography process. Ph.D. thesis; The University of Texas at Austin; 2006.

[17] Taylor, H., Lam, Y.C., Boning, D.. A computationally simple method for simulating the micro-embossing of thermoplastic layers. Journal of Micromechanics and Microengineering 2009;19(7):075007-16.

[18] Chauhan, S., Palmieri, F., Bonnecaze, R.T., Willson, C.G.. Feature filling modeling for step and flash imprint lithography. Journal of Vacuum Science Technology B: Microelectronics and Nanometer Structures 2009;27(4):1926 -1932. doi:10.1116/1.3147212.

[19] Reynolds, O.. On the theory of lubrication and its application to Mr. Beauchamp Tower's experiments, including an experimental determination of the viscosity of olive oil. Philosophical Transactions of the Royal Society of London 1886;177:157-234.

[20] Osher, S.J., Fedkiw, R.P.. Level Set Methods and Dynamic Implicit Surfaces. Springer; 2002.

[21] Francois, M.M., Cummins, S.J., Dendy, E.D., Kothe, D.B., Sicilian, J.M., Williams, M.W.. A balanced-force algorithm for continuous and sharp interfacial surface tension models within a volume tracking framework. Journal of Computational Physics 2006;213(1):141 - 173. doi:10.1016/j.jcp.2005.08.004. 
[22] Herrmann, M.. A balanced force refined level set grid method for two-phase flows on unstructured flow solver grids. Journal of Computational Physics 2008;227(4):2674 2706. doi:10.1016/j.jcp.2007.11.002.

[23] van Genuchten, M.T.. A closed-form equation for predicting the hydraulic conductivity of unsaturated soils. Soil Sci Soc Am J 1980;44:892-898. doi:10.2136/sssaj1980.03615995004400050002x.

[24] Forchheimer, P.. Wasserbewegung durch boden. Zeitschrift Verein Deutscher Ingenieure 1901;45:1782-1788.

[25] Brinkman, H.. A calculation of the viscous force exerted by a flowing fluid on a dense swarm of particles. Applied Scientific Research 1949;1(1):27-34. doi:10.1007/BF02120313.

[26] de Nevers, N.. Fluid Mechanics for Chemical Engineers. 2nd ed.; McGraw Hill; 1991.

[27] Panton, R.L.. Incompressible Flow. 3rd ed.; Wiley; 2005.

[28] Martic, G., Gentner, F., Seveno, D., Coulon, D., De Coninck, J., Blake, T.D.. A molecular dynamics simulation of capillary imbibition. Langmuir 2002;18(21):7971-7976. doi:10.1021/la020068n. arXiv:http://pubs . acs .org/doi/pdf/10.1021/la020068n .

[29] Blake, T.D., De Coninck, J.. The influence of solid-liquid interactions on dynamic wetting. Advances in Colloid and Interface Science 2002;96(1-3):21 -- 36. doi:10.1016/S0001-8686(01)00073-2.

[30] Blake, T., De Coninck, J.. The influence of pore wettability on the dynamics of imbibition and drainage. Colloids and Surfaces A: Physicochemical and Engineering Aspects 2004;250(1-3):395 -- 402. doi:10.1016/j.colsurfa.2004.05.024.

[31] Sangani, A.S., Acrivos, A.. Slow flow past periodic arrays of cylinders with application to heat transfer. International Journal of Multiphase Flow 1982;8(3):193 -- 206. doi:10.1016/0301-9322(82)90029-5. 
[32] Drummond, J., Tahir, M.. Laminar viscous flow through regular arrays of parallel solid cylinders. International Journal of Multiphase Flow $1984 ; 10(5): 515$-- 540. doi:10.1016/0301-9322(84)90079-X.

[33] Cairncross, R.A., Schunk, P.R., Chen, K.S., Prakash, S.S., Samuel, J., Hurt, A.J., et al. Drying in deformable partially-saturated porous media: Sol-gel coatings. Tech. Rep. SAND96-2149; Sandia National Laboratories; 1996.

[34] Schunk, P.R., Sackinger, P.A., Rao, R.R., Chen, K.S., Baer, T., Labreche, D.A., et al. GOMA 5.0 - a full-Newton finite element program for free and moving boundary problems with coupled fluid/solid momentum, energy, mass, and chemical species transport: User's guide. Tech. Rep. SAND2006-5375; Sandia National Laboratories; 2006.

[35] Heroux, M., Bartlett, R., Hoekstra, V.H.R., Hu, J., Kolda, T., Lehoucq, R., et al. An Overview of Trilinos. Tech. Rep. SAND2003-2927; Sandia National Laboratories; 2003. 

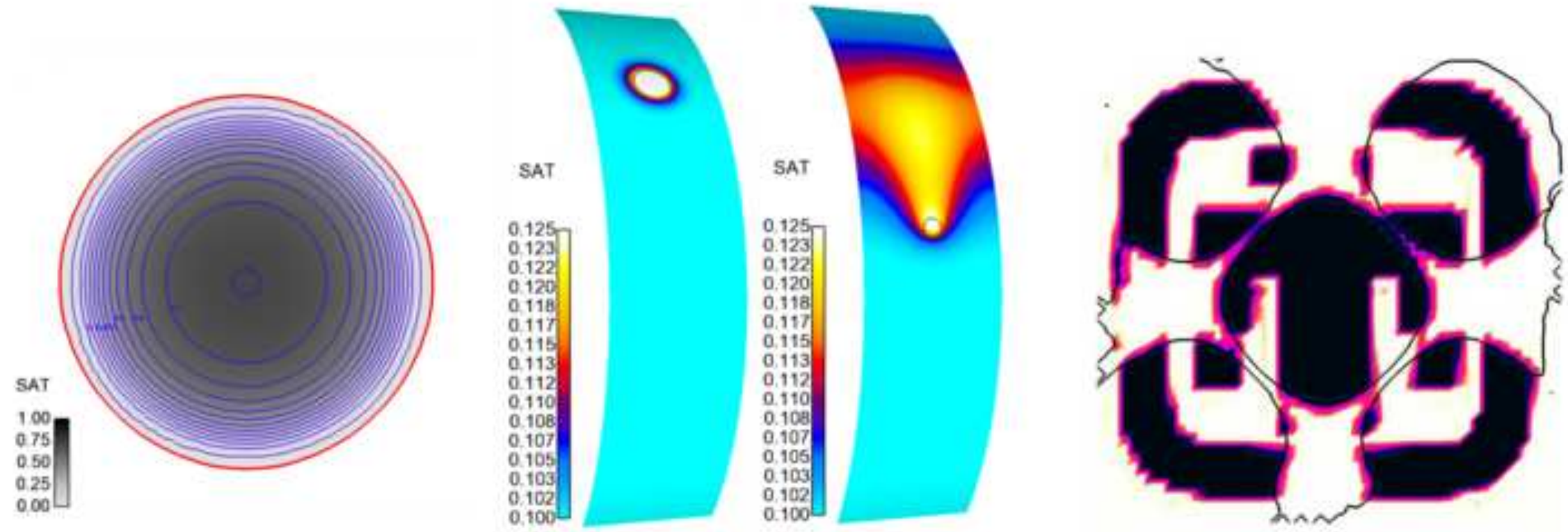

Saturation of various structured and unstructured thin porous materials 